\title{
Structural and dynamic basis for evolutionary bifurcations in enzyme families Colin Jackson ${ }^{1}$, Eleanor CampbelI ${ }^{2}$, Charlotte Miton ${ }^{3}$, Nobuhiko Tokuriki ${ }^{4}$ ${ }^{1}$ Australian National University ${ }^{2}$ Australian Synchrotron, ${ }^{3}$ University British Columbia, ${ }^{4}$ University British Columbia colin.jackson@anu.edu.au
}

The bifurcation of evolutionary pathways, in which a trajectory separates and produces two eventually noninterchangeable trajectories, is the basis of diversification, from higher organisms to proteins. Here, we present a detailed molecular picture of the events involved in a bifurcation event that led to the emergence of two distinct enzymes from a common ancestor. Laboratory evolution of a phosphotriesterase, PTE, toward arylesterase activity, resulted in dramatically different genetic and phenotypic outcomes when evolved from two starting points that differed by a single amino acid (Arg254 vs. Ser254). One trajectory led to a $>70,000$-fold increase in esterase activity by reorganizing the active site architecture to be highly complementary to the new substrate. In contrast, the other only resulted in a $\sim 500$ fold increase, through the introduction of structural disorder in a key active site loop that created an alternative substrate binding mode. The initial changes (Arg254 vs. Ser254) are sufficiently different that unique structural changes must be made, i.e. what works with one does not with with the other. We also observe interesting crystallographic artefacts that result from an active site loop becoming functionally redundant and highly dynamic, whereby it reaches into a neighbouring active site and results in a novel trinuclear $\mathrm{Zn} 2+$ active site. 